\title{
BLUNT CEREBROVASCULAR INJURIES
}

\author{
C. Clay Cothren, and Ernest E. Moore
}

Cothren CC, Moore EE. Blunt cerebrovascular injuries. Clinics. 2005;60(6):489-96.

Over the past decade, the recognition and subsequent management of blunt cerebrovascular injuries has undergone a marked evolution. Originally thought to be a rare occurrence, blunt cerebrovascular injuries are now diagnosed in approximately $1 \%$ of blunt trauma patients. The recognition of a clinically silent period allows for angiographic screening for injuries based upon the mechanism of trauma and the patient's constellation of injuries. Comprehensive screening of patients has resulted in the early diagnosis of blunt cerebrovascular injuries during the asymptomatic phase, thus allowing treatment that could prevent neurologic sequelae. Although the ideal regimen of antithrombotic therapy is yet to be determined, treatment with either antiplatelet or anticoagulant agents has been shown to reduce the blunt cerebrovascular injuries related stroke rate. Blunt cerebrovascular injury is a rare but potentially devastating injury; appropriate angiographic screening in high-risk patients should be performed and prompt treatment initiated to prevent ischemic neurologic events.

\section{KEYWORDS: Artery. Carotid. Blunt. Cerebrovascular. Stroke. Injury. Vertebral.}

Over the past decade, a wealth of studies has provided the scientific rationale for promoting the early screening and treatment of blunt cerebrovascular injuries (BCVI). Initially, BCVI were thought to have unavoidable, devastating neurologic outcomes, but several reports have suggested that anticoagulant therapy improves neurologic outcome in patients suffering ischemic neurologic events. ${ }^{1-7}$ If untreated, carotid artery injuries (CAI) have a stroke rate up of to $50 \%$ depending on the injury grade, with increasing stroke rates correlated with increasing grades of injury; vertebral artery injuries (VAI) have a stroke rate of $25 \% .^{3}$ Because many of the patients with BCVI have concomitant traumatic brain injuries, poor outcomes due to neurologic sequelae to BCVI have been attributed to the traumatic brain injury. Screening protocols based on patient injury patterns and mechanism of injury have been instituted prior to neurologic sequelae to identify these injuries in asymptomatic patients and to initiate stroke-preventative treatment. Current studies suggest that early antithrombotic therapy in patients

Department of Surgery, Denver Health Medical Center - Denver/CO, USA. University of Colorado Health Sciences Center - Denver/CO, USA.

Email: clay.cothren@dhha.org

Received for publication on July 25, 2005.

Accepted for publication on September 09, 2005. with BCVI reduces stroke rates and prevents neurologic morbidity. ${ }^{1,3,4,8-11}$

\section{Historical Perspective}

Blunt cerebrovascular injuries were first recognized more than 30 years ago, ${ }^{12}$ but the majority of patients with BCVI presented with symptoms of neurologic ischemia. ${ }^{12-16}$ The patient's presenting symptom of cerebral ischemia may reveal the underlying cerebrovascular injury. Carotid artery injuries generally result in contralateral sensorimotor deficits, while VAIs typically manifest as ataxia, dizziness, vomiting, facial or body analgesia, or visual field defects. Aphasia may be present if the dominant hemisphere is involved, while nondominant hemisphere strokes may result in hemineglect. Symptoms of carotid-cavernous fistulae include orbital pain, exophthalmos, chemosis, and conjunctival hyperemia.

Crissey and Bernstein postulated 3 fundamental mechanisms of injury: direct blow to the neck, hyperextension with contralateral rotation of the head, and laceration of the artery by adjacent fractures involving the sphenoid or petrous bones. ${ }^{12}$ The most common mechanism causing CAI is hyperextension resulting from the stretching of the 
carotid artery over the lateral articular processes of $\mathrm{C} 1$ C3. ${ }^{13-18}$ Vertebral artery injuries are likely a combination of direct injury, due to associated fractures of the vertebrae involving the transverse foramen through which the artery courses, and hyperextension-stretch injury due to the tethering of the vertebral artery within the lateral masses of the cervical spine. Regardless of mechanism, there is intimal disruption of the carotid or vertebral artery. This intimal tear becomes a nidus for platelet aggregation that may lead to emboli or vessel occlusion.

Following the recognition that BCVI were responsible for adverse neurologic events in some patients, treatment modalities were debated. The vast majority of these lesions occur in surgically inaccessible areas of the blood vessels, either high within the carotid canal at the base of the skull or within the foramen transversarium. Such a location makes the standard vascular repair approaches including reconstruction or thrombectomy challenging if not impossible. Heparin was initially the treatment of choice for BCVI, with the assumption that this promoted clot stabilization if present and clot resolution through intrinsic fibrinolytic mechanisms, and it prevented further thrombosis. ${ }^{5,7,8}$ Treatment with anticoagulant agents has been shown to improve neurologic outcome in patients sustaining BCVI-related ischemic neurologic events (INE). ${ }^{5,7-9}$ Initial reports, including one of a multicenter study by the Western Trauma Association, showed that patients who were treated with anticoagulant agents had improved outcome compared to those who were either not treated or had a contraindication for anticoagulation due to associated head injuries. ${ }^{5,7}$ In these studies, up to $45 \%$ of patients achieved good neurologic status. The first single-institution study with a large volume of experience demonstrated heparin therapy was independently associated with survival and improvement in neurologic outcomes. ${ }^{8}$ Follow-up evaluations by other institutions support the use of anticoagulation after the onset of neurologic symptoms to improve overall functional outcome. ${ }^{4,9,19}$ Although the initial focus of BCVI was recognizing the injury and treating the devastating neurologic sequelae, subsequent efforts have been directed at diagnosing and treating these injuries during the "silent period," prior to the onset of stroke.

\section{BCVI Screening during the "Silent Period"}

Although some patients may present with symptoms within an hour of injury, the majority exhibit a latent period. This asymptomatic phase has been inferred based upon the time to onset of symptoms in patients who did not receive antithrombotic therapy. This time frame appears to range from hours up to 14 years, but the majority of patients seem to develop symptoms within 10 to 72 hours. ${ }^{3-5,17,18,20,21}$ Diagnosing BCVI during this "silent period" affords the opportunity for treatment prior to neurologic sequelae.

Aggressive screening for BCVI was initially suggested in the mid-1990s $\mathrm{s}^{8,9}$ after recognition that specific patterns of injuries were associated. ${ }^{5,722}$ Although optimal screening criteria are yet to be defined, current algorithms include patients with signs or symptoms, as well as those considered at high risk by the injury pattern ${ }^{1,4,11,18}$ (Table 1 ). A recently published report questioned the utility of such an aggressive screening approach, ${ }^{23}$ while other studies have reported a screening yield of over $30 \%$ in high-risk populations..$^{1,2,4,11,24}$

Table 1- Denver screening criteria for blunt cerebrovascular injury (BCVI)

\section{Signs/Symptoms of BCVI}

Arterial hemorrhage

Cervical bruit in patient $<50$ years of age

Expanding cervical hematoma

Focal neurologic deficit

Neurologic exam incongruous with head CT scan findings

Stroke on secondary CT scan

Risk Factors for BCVI

High-energy transfer mechanism with:

LeForte II or III fracture

Cervical-spine fracture patterns: subluxation, fractures extending into the transverse foramen, fractures of $\mathrm{C} 1-\mathrm{C} 3$

Basilar skull fracture with carotid canal involvement

Diffuse axonal injury with a Glascow Coma Scale (GCS) score $<6$

Near hanging with anoxic brain injury

Table 2 - Denver grading scale for blunt cerebrovascular injuries

Grade I: irregularity of the vessel wall or a dissection/intramural hematoma with less than $25 \%$ luminal stenosis

Grade II: intraluminal thrombus or raised intimal flap is visualized, or dissection/intramural hematoma with $25 \%$ or more luminal narrowing

Grade III: pseudoaneurysm

Grade IV: vessel occlusion

Grade V: vessel transection

\section{Diagnostic Imaging and Injury Grading Scale}

Until the sensitivity and specificity of computed tomographic angiography (CTA) or magnetic resonance angiography (MRA) approaches that of angiography, 4-vessel arteriography remains the gold standard for diagnosing BCVI. Undoubtedly, many clinicians question the need for subjecting patients to angiography. Angiography is labor intensive, costly, and not without risks; additionally, if not available at smaller hospitals, it requires emergent transfer of a pa- 
tient for definitive evaluation. Currently, CTA remains an unproven diagnostic modality for this injury, with a sensitivity of between $50 \%$ to $68 \% .^{2,3,25}$ In particular, injuries that may be missed by such noninvasive studies are typically grade I and II injuries; however, pseudoaneurysms and occlusions have also been misdiagnosed., ${ }^{2,3}$ The risk associated with angiography in our screened trauma population was $0.1 \%$, while the stroke risk for an undiagnosed grade I CAI is $8 \%$ and of VAI is $6 \%$. $^{3,4}$ While advances in technology with improved imaging by multislice $\mathrm{CT}$ scanners may become an alternative in the future, until these modalities are evaluated with follow-up angiography, standard 4-vessel angiography remains the standard of care.

All patients with indications for screening and no contraindications for antithrombotic therapy undergo angiography as soon as possible. In our institution, patients admitted during daytime hours undergo angiography prior to transport to the surgical intensive care unit from the emergency department unless an urgent operation intervenes; patients evaluated after daylight hours undergo angiography the morning following admission. If the patient is symptomatic on admission, emergent angiography is performed.

Along with the recognition of varied luminal irregularities comprising BCVI (dissection, occlusion, transection, and pseudoaneurysms) was the identification of disparate outcomes. ${ }^{5,8}$ An injury grading scale was developed ${ }^{18}$ to provide not only an accurate description of the injury, but also to define stroke risk by injury grade. Untreated injuries have an overall stroke rate of $21 \%$ to $64 \%,{ }^{1,11}$ CAIs have increasing stroke rate by increasing grade, and VAIs tend to have a more consistent stroke rate of approximately $20 \%$ for all grades of injury ${ }^{3}$ (Table 3 ). Figure 1 shows representative angiographic images of different grades of BCVI.

Patients undergo repeat arteriography 7 to 10 days after their initial diagnostic study. The importance of routine follow-up arteriography is particularly salient for patients with grade I and II injuries; over half of grade I injuries completely heal, allowing cessation of antithrombotic therapy. ${ }^{3}$ While only $8 \%$ of grade II injuries healed in this study, over $40 \%$ progressed to grade III injuries despite therapy; in patients with CAI, this increase in injury grade

Table 3 - Stroke rate by blunt cerebrovascular injury grade

\begin{tabular}{lcc}
\hline & Grade of injury & Stroke rate \\
\hline Carotid artery injuries & I & $3 \%$ \\
& II & $14 \%$ \\
& III & $26 \%$ \\
& IV & $50 \%$ \\
Vertebral artery injuries & V & $100 \%$ \\
& I & $6 \%$ \\
& II & $38 \%$ \\
& III & $27 \%$ \\
& IV & $28 \%$ \\
& V & $100 \%$ \\
\hline
\end{tabular}
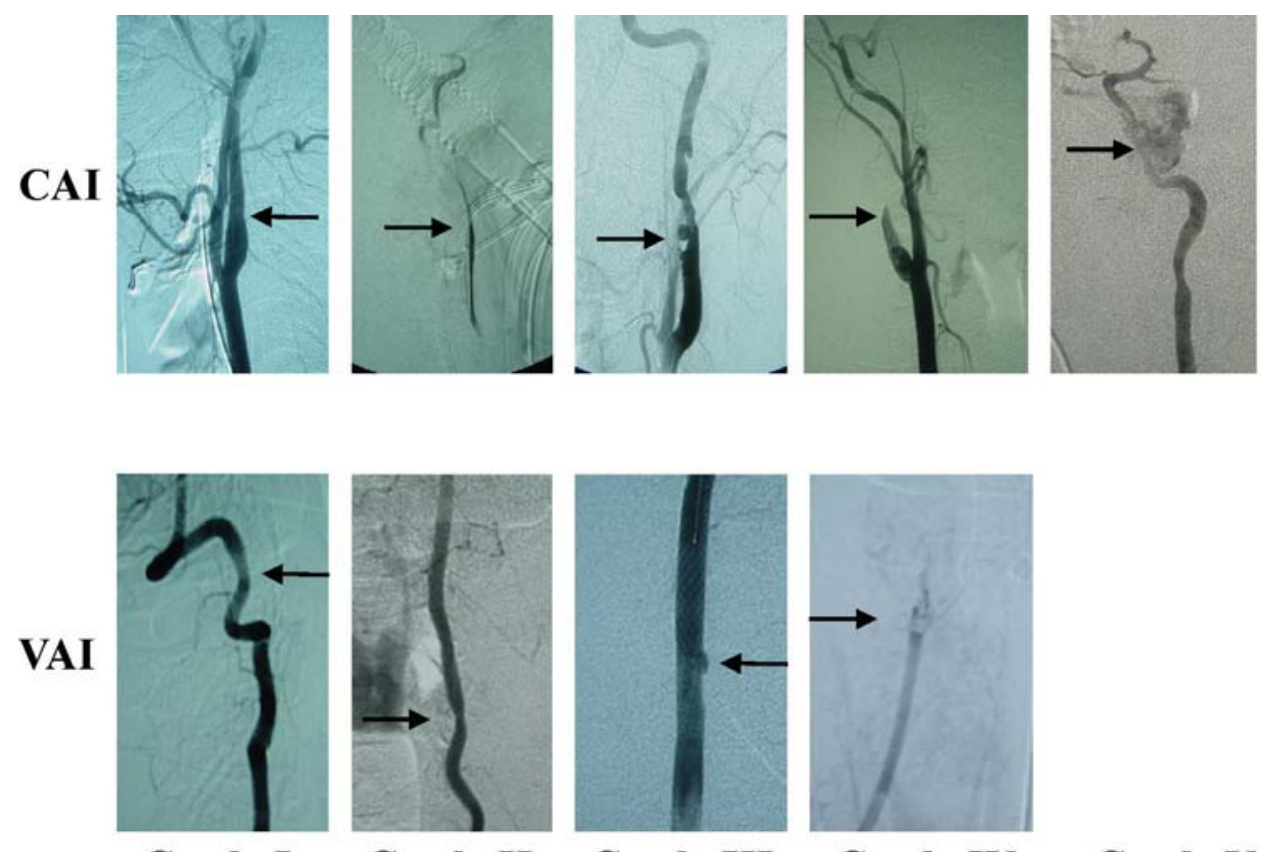

\section{Grade I Grade II Grade III Grade IV Grade V}

Figure 1 - Representative angiographic images of different grades of BCVI

Injury Grade: Angiographic Finding; Grade I: Intimal Irregularity, <25\% Luminal Stenosis; Grade II: Intimal Irregularity, $>25 \%$ Luminal Stenosis, Intimal Flap; Grade III: Pseudoaneurysm; Grade IV: Occlusion; Grade V: Transection with active extravasation. 
also correlates with an increase in stroke risk. Some authors have advocated an endovascular approach to pseudoaneurysms, hence supporting the use of repeat angiography for diagnosis of such lesions. Our most recent evaluation of endovascular stents in patients with postinjury BCVI, however, suggests that antithrombotic therapy remains the gold standard treatment. ${ }^{10}$ Patients with carotid or vertebral artery occlusions may not require reimaging, as $82 \%$ showed no change on follow-up imaging. ${ }^{3}$

\section{Treatment: Antithrombotics}

Initially, therapy for BCVI was based on anecdotal reports of neurologic improvement with heparinization in patients suffering stroke related to BCVI. ${ }^{5,7,8}$ Subsequently, intravenous heparin was thought to be the treatment of choice for those asymptomatic patients with blunt injuries. $^{2,3}$ Initially, standard heparinization protocols were used, but due to a moderate incidence of bleeding in multisystem trauma patients, we modified our protocol. ${ }^{9,18}$ Currently, anticoagulation with systemic heparin is initiated using a continuous infusion of heparin at $15 \mathrm{U} / \mathrm{kg} / \mathrm{h}$, without a loading dose; heparin drips are titrated to achieve a partial thromboplastin time (PTT) of between 40 and 50 seconds. With this adjustment in our BCVI heparin protocol, fewer than $1 \%$ of patients have had bleeding complications necessitating transfusion. ${ }^{4}$ For patients with contraindications for heparin, the initiation of antiplatelet agents (aspirin 325 $\mathrm{mg} / \mathrm{d}$ and clopidogrel $75 \mathrm{mg} / \mathrm{d}$ ) has gained favor. ${ }^{3,21,26} \mathrm{It}$ should be noted that antithrombotic therapy is not started with patients having closed head injury or intraparenchymal hemorrhage without input from the neurosurgery service.

Admittedly, there is controversy regarding the ideal antithrombotic therapy-anticoagulant versus antiplatelet agents - for any type of arterial disease. ${ }^{27-29} \mathrm{~A}$ retrospective study by Chimowitz et $\mathrm{al}^{30}$ indicated that warfarin is superior in patients with vertebrobasilar occlusive disease, while a more recent prospective double-blind comparison by the same authors demonstrated aspirin is the therapy of choice for patients with symptomatic intracranial atherosclerotic arterial stenosis, due to equivalent stroke prevention rates as for warfarin, but decreased hemorrhagic complications. ${ }^{30} \mathrm{~A}$ recent review of vertebrobasilar disease supported the use of antiplatelet agents in patients with arterial stenosis but warfarin in patients with severe, flow-limiting lesions or dissections. ${ }^{31}$ The selection of which therapeutic agent should be used and whether the choice of antithrombotic should be determined by the patient's injury grade must continue to be evaluated in prospective studies.

Most importantly, patients who are diagnosed early and treated with antithrombotics almost universally avoid INE. ${ }^{2,4}$
The Memphis group showed a reduction in stroke rate for CAI from $64 \%$ in untreated patients to $6.8 \%$ in treated patients (either with anticoagulant or antiplatelet agents), and for VAI a reduction in stroke rate from $54 \%$ to $2.6 \%$ in untreated versus treated patients. Our group's most recent evaluation demonstrated a stroke rate of $0.5 \%$ in 187 patients with BCVI treated with antithrombotics, while untreated patients had an overall stroke rate of $21 \% .{ }^{10}$ Although the optimal regimen remains undetermined, there appears to be equivalence between the two therapies. ${ }^{2-4}$ To determine the ideal therapeutic regimen for BCVI, we are currently enrolling patients in a prospective randomized study comparing antiplatelet agents to intravenous heparin in patients with grades I to III injuries. With an attendant permanent neurologic morbidity of up to $80 \%$ and mortality rates of up to $40 \%,{ }^{20,32,33}$ prompt treatment of diagnosed injuries is critical.

\section{Treatment: Endovascular Stents}

Over the past decade, there has been an explosion in the use of percutaneous transluminal arterial interventions for both traumatic injuries and atherosclerotic lesions. Although the role of carotid stents for atherosclerotic disease is being explored with randomized, well-controlled trials, ${ }^{34-37}$ the indication for percutaneous intervention for traumatic injuries is less well defined. Carotid stents have been utilized in patients with blunt injury with persistent pseudoaneurysms because of concern about subsequent embolization or rupture. ${ }^{38,39}$ In theory, the uncovered carotid stent acts as a filter to trap any thrombus within the pseudoaneurysm, thereby preventing subsequent embolization and stroke. The stent may also decrease flow into the pseudoaneurysm by increasing laminar flow within the stented portion of the carotid lumen itself. ${ }^{40}$ Decreasing flow into the aneurysmal sac may then reduce any egress of blood from the sac, which in turn may reduce turbulence within the lumen.

Several isolated case reports have advocated the use of percutaneous angioplasty and stenting of carotid injures. ${ }^{41-47}$ Not surprisingly, these case reports represent a diverse range of pathologies, symptoms, mechanisms of injury, and time to diagnosis. Although the majority appears to have patency of the stented carotid artery documented in followup radiographic evaluations, several cases of carotid artery occlusion following stent placement have been reported. ${ }^{42,44}$ Our most recent evaluation indicates a prohibitive stroke and carotid occlusion rate associated with carotid stents placed in acutely injured vessels ${ }^{11}$; additionally, long-term follow-up in patients with traumatic pseudoaneurysms who were treated with anticoagulant therapy alone has not been evaluated. Further understanding and evaluation of the role of appropriate concurrent antithrombotic therapy, as well 
as evolving stent technology including smaller delivery systems and covered stents, may improve the outcome for postinjury intraluminal carotid stents. In the interim, however, our experience to date suggests that carotid stenting should be performed in selective cases, and antithrombotic therapy should remain the cornerstone of treatment for posttraumatic pseudoaneurysms.

\section{Long-Term Treatment and Outcomes}

Following initiation of antithrombotics, treatment is empirically continued for 6 months. Our current protocol is to transition the patient to warfarin if the initial antithrombotic therapy was heparin, with a goal INR (international normalized ratio) of 2.0. If the patient was started on antiplatelet agents, these are continued after hospital discharge. Although complete healing of grade I injuries on repeat angiography at 7-10 days has been documented in more than half of affected patients, the vast majority of grade II, III, and IV injuries persist. Comprehensive long-term follow-up beyond the acute hospitalization has not been reported in the literature, as is true in most trauma population studies. Therefore, whether these injuries heal or persist at 3 to 6 months is unknown. There are anecdotal reports of carotid pseudoaneurysm rupture, particularly in the petrous portion of the canal leading to epistaxis. ${ }^{48-53}$ However, aside from these limited cases located intracranially, few other reports are evident in the literature. ${ }^{54,55}$ With so little long-term data, it is difficult to ascertain either the true healing rate of these injuries or the risk of rupture or embolic stroke.

The morbidity and mortality of BCVI-related INEs is well documented. Stroke-related need for discharge and overall rate of discharge to rehabilitation services is higher in patients suffering BCVI-related INE. ${ }^{10}$ Such prolonged acute patient care increases costs to patients, to insurance companies, and ultimately to society. Although delineating the precise BCVI-related costs within a patient's acute or rehab stay is difficult in multisystem trauma patients, calculating the cost of a patient's life is even more problematic. is even more problematic. Mortality due to BCVI is significant, with CAI patients having a $13 \%$ to $21 \%$ stroke-related mortality and patients with VAI-related strokes having a $4 \%$ to $18 \%$ mortality rate in modern series. ${ }^{1,11}$ The impact on mortality due to BCVI-related strokes appears independent of the patient's associated injuries. Overall mortality in patients sustaining CAI was $7 \%$ for those without a neurologic event versus $32 \%$ for those with a neurologic event; in patients with VAI, those without neurologic event had a mortality of $7 \%$ while those with a neurologic event had a mortality rate of $18 \%$; the ISS (illness severity scale) was not significantly different between those with and without INE. ${ }^{10}$

Repeat evaluation of the patient's injury and a determination of antithrombotic therapy should be considered at 6 months. Although no long-term studies have been performed to date, we currently recommend CTA for patients with initially diagnosed grade III and IV injuries, and angiography for grade I and II injuries. If the patient does not wish to undergo angiography with its attendant risks, we empirically consider placing the patient on life-long aspirin. Similarly, patients with persistent grade III and IV injuries on repeat imaging are empirically continued on life-long aspirin.

\section{CONCLUSIONS}

Diagnosis and treatment of blunt cerebrovascular injuries has evolved over the past 3 decades. Currently, protocols exist for establishing an indication for angiographic screening based on the injury mechanism and associated injuries, ${ }^{7}$ hence limiting invasive procedures to those with the highest risk of injury (Figure 2). Following identification of injuries in asymptomatic patients, prompt initiation of antithrombotic therapy reduces the incidence of ischemic neurologic events. Surgeons caring for the multiply injured should screen for carotid and vertebral artery injuries in high-risk patients.

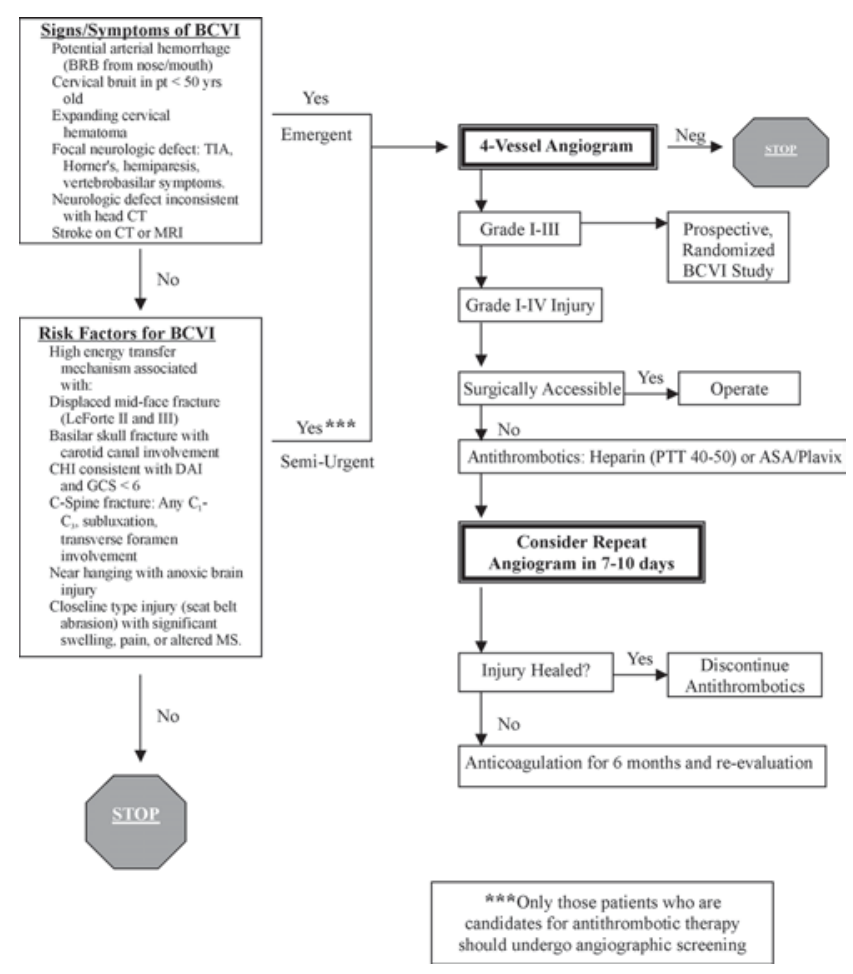

Figure 2 - Denver screening and treatment algorithm for BCVI 
Cothren CC, Moore EE. Traumatismo cerebrovascular contuse. Clinics. 2005;60(6):489-96.

Durante a década passada, o reconhecimento e tratamento do traumatismo cerebrovascular contuso, sofreu importante evolução. Este tipo de ferimento era considerado como ocorrência rara, mas atualmente o quadro é diagnosticado em cerca de $1 \%$ dos pacientes. O reconhecimento da existência de um período clínico silencioso permite uma seleção angiográfica baseada no mecanismo de trauma e na sistematização dos ferimentos dos pacientes.

A avaliação sistemática e a suspeita diagnóstica precoce destes pacientes tem resultado em rápido confirmação durante a fase assintomática, permitindo a instauração de tra- tamento cuja meta é impedir o desenvolvimento de seqüelas neurológicas. Embora o tratamento ideal, antitrombótico, ainda precise ser determinado, o uso de agentes antiplaquetários ou anticoagulantes reduz a incidência de lesões cerebrovasculares relacionada a fenômenos tromboembolíticos.

O traumatismo cerebrovascular contuso é raro, porém devastador. A seleção angiográfica apropriada em pacientes de alto risco deve ser realizada e o tratamento deve ser imediatamente iniciado para impedir eventos neurológicos isquêmicos.

PAlAVRAS-CHAVE: Artéria. Carótida. Contuso. Cerebrovascular. Infarto. Ferimento. Vertebral.

\section{REFERENCES}

1. Miller PR, Fabian TC, Bee TK, Timmons S, Chamsuddin A, Finkle R, et al. Blunt cerebrovascular injuries: diagnosis and treatment. J Trauma. 2001;51:279-85.

2. Miller PR, Fabian TC, Croce MA, Cagiannos C, Williams JS, Vang M, et al. Prospective screening for blunt cerebrovascular injuries: analysis of diagnostic modalities and outcomes. Ann Surg. 2002;236:386-93.

3. Biffl WL, Ray CE Jr, Moore EE, Franciose RJ, Aly S, Heyrosa MG, et al. Treatment-related outcomes from blunt cerebrovascular injuries: importance of routine follow-up arteriography. Ann Surg. 2002;235:699706; discussion 706-7.

4. Cothren CC, Moore EE, Biffl WL, Ciesla DJ, Ray CE Jr, Johnson JL, et al. Anticoagulation is the gold standard therapy for blunt carotid injuries to reduce stroke rate. Arch Surg. 2004;139:540-5.

5. Cogbill TH, Moore EE, Meissner M, Fischer RP, Hoyt DB, Morris JA, et al. The spectrum of blunt injury to the carotid artery: a multicenter perspective. J Trauma. 1994;37:473-9.

6. Anson J, Crowell RM. Cervicocranial arterial dissection. Neurosurgery. 1991;29:89-96

7. Davis JW, Holbrook TL, Hoyt DB, Mackersie RC, Field TO Jr, Shackford SR. Blunt carotid artery dissection: incidence, associated injuries, screening, and treatment. J Trauma. 1990;30:1514-7.

8. Fabian TC, Patton JH Jr, Croce MA, Minard G, Kudsk KA, Pritchard FE. Blunt carotid injury. Importance of early diagnosis and anticoagulant therapy. Ann Surg. 1996;223:513-22.
9. Biffl WL, Moore EE, Ryu RK, Offner PJ, Novak Z, Coldwell DM, et al. The unrecognized epidemic of blunt carotid arterial injuries: early diagnosis improves neurologic outcome. Ann Surg. 1998;228:462-70.

10. Cothren CC, Moore EE, Ray CE. Screening for blunt cerebrovascular injuries is cost effective. Am J Surg. In press.

11. Cothren CC, Moore EE, Ray CE, et al. Carotid artery stents for blunt cerebrovascular injury: Risks exceed benefits. Arch Surg. 2005;140: 480-6.

12. Crissey MM, Bernstein EF. Delayed presentation of carotid intimal tear following blunt craniocervical trauma. Surgery. 1974;75:543-9.

13. Batzdorf U, Bentson JR, Machleder HI. Blunt trauma to the high cervical carotid artery. Neurosurgery. 1979;5:195-201.

14. Perry MO, Snyder WH, Thal ER. Carotid artery injuries caused by blunt trauma. Ann Surg. 1980;192:74-7.

15. Dragon R, Saranchak H, Lakin P, Strauch G. Blunt injuries to the carotid and vertebral arteries. Am J Surg. 1981;141:497-500.

16. Welling RE, Saul TG, Tew JM Jr, Tomsick TA, Kremchek TE, Bellamy MJ. Management of blunt injury to the internal carotid artery. J Trauma. 1987;27:1221-6

17. Mokri B, Piepgras DG, Houser OW. Traumatic dissections of the extracranial internal carotid artery. J Neurosurg. 1988;68:189-97. 
18. Biffl WL, Moore EE, Offner PJ, Brega KE, Franciose RJ, Burch JM. Blunt carotid arterial injuries: implications of a new grading scale. $\mathrm{J}$ Trauma. 1999;47:845-53.

19. Eachempati SR, Vaslef SN, Sebastian MW, Reed RL, 2nd. Blunt vascular injuries of the head and neck: is heparinization necessary? J Trauma. 1998;45:997-1004.

20. Krajewski LP, Hertzer NR. Blunt carotid artery trauma: report of two cases and review of the literature. Ann Surg. 1980;191:341-6.

21. Fabian TC, George SM Jr, Croce MA, Mangiante EC, Voeller GR, Kudsk KA. Carotid artery trauma: management based on mechanism of injury. J Trauma. 1990;30:953-61; discussion 961-3.

22. Parikh AA, Luchette FA, Valente JF, Johnson RC, Anderson GL, Blebea J, et al. Blunt carotid artery injuries. J Am Coll Surg. 1997;185:80-6.

23. Mayberry JC, Brown CV, Mullins RJ, Velmahos GC. Blunt carotid artery injury: the futility of aggressive screening and diagnosis. Arch Surg. 2004;139:609-12; discussion 612-3.

24. Biffl WL, Moore EE, Offner PJ. Optimizing screening for blunt cerebral vascular injuries. Am J Surg. 1999;178:517-522.

25. Wahl WL, Brandt MM, Thompson BG, Taheri PA, Greenfield LJ. Antiplatelet therapy: an alternative to heparin for blunt carotid injury. J Trauma. 2002;52:896-901.

26. Wahl WL, Ahrns KS, Brandt MM, Franklin GA, Taheri PA. The need for early angiographic embolization in blunt liver injuries. J Trauma. 2002;52:1097-101.

27. Johnson WC, Williford WO. Benefits, morbidity, and mortality associated with long-term administration of oral anticoagulant therapy to patients with peripheral arterial bypass procedures: a prospective randomized study. J Vasc Surg. 2002;35:413-21.

28. Oostenbrink JB, Tangelder MJ, Busschbach JJ, van Hout BA, Buskens E, Algra A, et al. Cost-effectiveness of oral anticoagulants versus aspirin in patients after infrainguinal bypass grafting surgery. J Vasc Surg. 2001;34:254-62.

29. Tangelder MJ, Algra A, Lawson JA, Hennekes S, Eikelboom BC. Optimal oral anticoagulant intensity to prevent secondary ischemic and hemorrhagic events in patients after infrainguinal bypass graft surgery. Dutch BOA Study Group. J Vasc Surg. 2001;33:522-7.

30. Chimowitz MI, Kokkinos J, Strong J, Brown MB, Levine SR, Silliman $\mathrm{S}$, et al. The Warfarin-Aspirin Symptomatic Intracranial Disease Study. Neurology. 1995;45:1488-93.

31. Savitz SI, Caplan LR. Vertebrobasilar disease. N Engl J Med. 2005;352:2618-26.

32. Fakhry SM, Jaques PF, Proctor HJ. Cervical vessel injury after blunt trauma. J Vasc Surg. 1988;8:501-8.

33. Martin RF, Eldrup-Jorgensen J, Clark DE, Bredenberg CE. Blunt trauma to the carotid arteries. Vascular Surgery. 1991;14:789-93 (discussion 793-5).

34. CAVATAS. Endovascular versus surgical treatment in patients with carotid stenosis in the Carotid and Vertebral Artery Transluminal Angioplasty Study (CAVATAS): a randomized trail. Lancet. 2001;357:1729-37.
35. Yadav JS. The 12-month SAPPHIRE results. Paper presented at: Annual Meeting of Transcatheter Cardiovascular Therapeutics; Sept 15-19, 2003; Washington, DC.34.

36. Wholey MH. Unanswered issues for carotid stenting in 2003. Catheter Cardiovasc Interv. 2003;60:570-2.

37. Eskandari MK, Longo GM, Vijungco JD, Morasch MD, Pearce WH. Does carotid stenting measure up to endarterectomy? A vascular surgeon's experience. Arch Surg. 2004;139:734-8.

38. Coldwell DM, Novak Z, Ryu RK, Brega KE, Biffl WL, Offner PJ, et al. Treatment of posttraumatic internal carotid arterial pseudoaneurysms with endovascular stents. J Trauma. 2000;48:470-2.

39. Houry D, Colwell C, Ott C. Abdominal pain in a child after blunt abdominal trauma: an unusual injury. J Emerg Med. 2001;21:239-41.

40. Lu CJ, Kao HL, Sun Y, Liu HM, Jeng JS, Yip PK, et al. The hemodynamic effects of internal carotid artery stenting: a study with color-coded duplex sonography. Cerebrovasc Dis. 2003;15:264-9.

41. Kerby JD, May AK, Gomez CR, Rue LW, 3rd. Treatment of bilateral blunt carotid injury using percutaneous angioplasty and stenting: case report and review of the literature. J Trauma. 2000;49:784-7.

42. Duane TM, Parker F, Stokes GK, Parent FN, Britt LD. Endovascular carotid stenting after trauma. J Trauma. 2002;52:149-53.

43. Shames ML, Davis JW, Evans AJ. Endoluminal stent placement for the treatment of traumatic carotid artery pseudoaneurysm: case report and review of the literature. J Trauma. 1999;46:724-6.

44. Liu AY, Paulsen RD, Marcellus ML, Steinberg GK, Marks MP. Longterm outcomes after carotid stent placement treatment of carotid artery dissection. Neurosurgery. 1999;45:1368-73; discussion 1373-4.

45. Fusonie GE, Edwards JD, Reed AB. Covered stent exclusion of blunt traumatic carotid artery pseudoaneurysm: case report and review of the literature. Ann Vasc Surg. 2004;18:376-9.

46. Fanelli F, Salvatori FM, Ferrari R, Pacella S, Rossi P, Passariello R. Stent repair of bilateral post-traumatic dissections of the internal carotid artery. J Endovasc Ther. 2004;11:517-21.

47. Akiyama Y, Nakahara I, Tanaka M, Iwamuro Y, Hayashi J, Harada K, et al. Urgent endovascular stent-graft placement for a ruptured traumatic pseudoaneurysm of the extracranial carotid artery. J Trauma. 2005;58:624-7.

48. Costantino PD, Russell E, Reisch D, Breit RA, Hart C.Ruptured petrous carotid aneurysm presenting with otorrhagia and epistaxis. Am J Otol. 1991;12:378-83.

49. Willinsky R, Lasjaunias P, Berenstein A. Intracavernous branches of the internal carotid artery (ICA). Comprehensive review of their variations. Surg Radiol Anat. 1987;9:201-15.

50. Chen D, Concus AP, Halbach VV, Cheung SW. Epistaxis originating from traumatic pseudoaneurysm of the internal carotid artery: diagnosis and endovascular therapy. Laryngoscope. 1998;108:326-31.

51. Han MH, Sung MW, Chang KH, Min YG, Han DH, Han MC. Traumatic pseudoaneurysm of the intracavernous ICA presenting with massive epistaxis: imaging diagnosis and endovascular treatment. Laryngoscope. 1994;104:370-7. 
52. Roth TC, Chaloupka JC, Putman CM, Ross DA, Weaver EM, Tarro J, et al. Percutaneous direct-puncture acrylic embolization of a pseudoaneurysm after failed carotid stenting for the treatment of acute carotid blowout. AJNR Am J Neuroradiol. 1998;19:912-6.

53. Auyeung KM, Lui WM, Chow LC, Chan FL. Massive epistaxis related to petrous carotid artery pseudoaneurysm after radiation therapy: emergency treatment with covered stent in two cases. AJNR Am J Neuroradiol. 2003;24:1449-52.
54. Tachibana E, Suzuki Y, Harada T, Saito K, Gupta SK, Yoshida J. Bypass surgery using a radial artery graft for bilateral extracranial carotid arteries occlusion. Neurosurg Rev. 2000;23:52-5.

55. Smith DC, Smith LL. Can carotid duplex scanning supplant arteriography in patients with focal carotid territory symptoms? J Vasc Surg. 1987;6:424-5. 